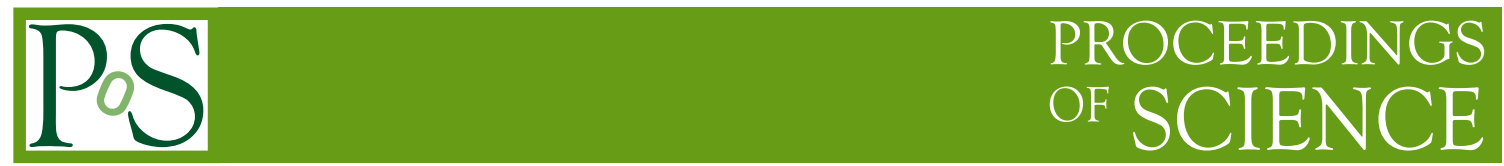

\title{
Long-baseline neutrino experiments
}

\section{Masashi Yokoyama*}

Department of Physics, Graduate School of Science, The University of Tokyo and Next-generation Neutrino Science Organization, The University of Tokyo and Kavli Institute for the Physics and Mathematics of the Universe (WPI), The University of Tokyo

E-mail: masashiephys.s.u-tokyo.ac.jp

Since the discovery 20 years ago, neutrino oscillations have been providing important new information for the particle physics. In this talk, recent results from reactor and accelerator based neutrino oscillation experiments are summarized in the context of standard three flavor framework.

The 39th International Conference on High Energy Physics (ICHEP2018)

4-11 July, 2018

Seoul, Korea

${ }^{*}$ Speaker. 


\section{Introduction}

This year marks the 20th anniversary of the discovery of neutrino oscillations by SuperKamiokande atmospheric neutrino observation [1]. Over the past two decades, various neutrino experiments provided us many surprises and discoveries. Studies of neutrino oscillation and neutrino properties have grown to be one of central topics in the particle physics. The tiny mass and large mixing of neutrinos imply the physics beyond Standard Model of particle physics. Properties of neutrinos are considered to be connected with fundamental questions, such as source of the baryon asymmetry of the universe, new physics law at the very high energy scale, and origin of fermion generations. In order to understand what neutrinos are telling us, we need more information on their properties and current and future experiments continue to explore the mysteries of neutrinos with improved precision and new set of measurements.

Currently, all of well-established experimental results can be consistently described by the framework of the standard three flavor neutrino mixing, where the mass and flavor eigenstates are related by the Maki-Nakagawa-Sakata (MNS) matrix (neglecting the Majorana phases which do not contribute to the neutrino oscillation):

$$
U=\left(\begin{array}{ccc}
1 & 0 & 0 \\
0 & \cos \theta_{23} & \sin \theta_{23} \\
0 & -\sin \theta_{23} & \cos \theta_{23}
\end{array}\right)\left(\begin{array}{cccc}
\cos \theta_{13} & 0 & \sin \theta_{13} e^{-\delta_{C P}} \\
0 & 1 & 0 \\
-\sin \theta_{13} e^{\delta_{C P}} & 0 & \cos \theta_{13}
\end{array}\right)\left(\begin{array}{ccc}
\cos \theta_{12} & \sin \theta_{12} & 0 \\
-\sin \theta_{12} & \cos \theta_{12} & 0 \\
0 & 0 & 1
\end{array}\right)
$$

If, and only if, the mixing angle $\theta_{i j}$ and the mass-squared difference $\Delta m_{i j}^{2}=m_{i}^{2}-m_{j}^{2}$ are both non-zero, the flavor of neutrinos change over their flight. This is the phenomena called neutrino oscillation. The probability of neutrino oscillation in vacuum, from a flavor $\alpha$ to $\beta$ with the neutrino energy $E$ and the flight length $L$, can be expressed as

$$
\begin{aligned}
P\left(v_{\alpha} \rightarrow v_{\beta}\right)=\delta_{\alpha \beta} & -4 \sum_{i>j} \mathfrak{R}\left(U_{\alpha i}^{*} U_{\beta i} U_{\alpha j} U_{\beta j}^{*}\right) \sin ^{2}\left(\frac{\Delta m_{i j}^{2} L}{4 E}\right) \\
& +2 \sum_{i>j} \mathfrak{I}\left(U_{\alpha i}^{*} U_{\beta i} U_{\alpha j} U_{\beta j}^{*}\right) \sin \left(\frac{\Delta m_{i j}^{2} L}{2 E}\right)
\end{aligned}
$$

Because of the small mass of neutrinos, a neutrino oscillation for energy of relevant experiments and observations, $\mathrm{MeV}$ to $\mathrm{GeV}$, happens with a flight length of $\mathrm{km}$ to $10,000 \mathrm{~km}$. It is a unique phenomenon in which a quantum effect manifest itself over a macroscopic scale.

The current generation of neutrino oscillation experiments have several major goals:

- More precise measurements of oscillation parameters

- Determination of mass hierarchy (ordering), i.e. whether $m_{3}$ is larger or smaller than $m_{1} \& m_{2}$

- Determination of $\theta_{23}$ octant, i.e. if $\theta_{23}$ is less than, greater than, or equal to $45^{\circ}$

- Search for CP violation in the lepton sector, and if discovered measurement of its amount

In this contribution, latest results from reactor- and accelerator-based long baseline neutrino experiments are summarized. We focus on the interpretation within the standard three flavor mixing framework. 


\section{Reactor neutrino experiments}

\subsection{Precision $\theta_{13}$ measurements}

Table 1 summarizes three reactor experiments designed for precise $\theta_{13}$ measurement $[2,3,4]$. They have different parameters, but all three experiments are based on the the same basic strategy.

Table 1: Parameters of three reactor $\theta_{13}$ experiments

\begin{tabular}{cccc}
\hline \hline & Daya Bay & Double Chooz & RENO \\
\hline \hline Reactor power $\left(\mathrm{GW}_{\text {th }}\right)$ & 17.4 & 8.5 & 16.8 \\
Baseline $(\mathrm{m})$ & $470 / 576 / 1650$ & $400 / 1050$ & $409 / 1444$ \\
Overburden: near/far (m.w.e.) & $250 / 265 / 860$ & $80 / 300$ & $120 / 450$ \\
Gd target mass for far detectors (tons) & 80 & 8.3 & 16.5 \\
\hline \hline
\end{tabular}

The survival probability for an electron anti-neutrino can be written as

$$
P\left(\bar{v}_{e} \rightarrow \bar{v}_{e}\right)=1-\sin ^{2} 2 \theta_{13} \sin ^{2}\left[\frac{\Delta m_{e e}^{2} L}{4 E}\right]-\cos ^{4} \theta_{13} \sin ^{2} 2 \theta_{12} \sin ^{2}\left[\frac{\Delta m_{21}^{2} L}{4 E}\right] .
$$

With a few $\mathrm{MeV}$ electron anti-neutrinos from reactors and at a distance of $\sim 1 \mathrm{~km}$ from the source, the second term of Eq. (2.1) becomes negligible and almost pure $\sin ^{2} 2 \theta_{13}$ measurement is possible. Electron anti-neutrinos are detected by inverse beta decay $\left(\bar{v}_{e}+p \rightarrow e^{+}+n\right)$ with the delayed coincidence technique.

In order to reduce systematic uncertainties, near and far detectors with identical design are used in all three experiments. The design of anti-neturino detectors is also basically common for three experiments. The central region contains Gd-doped liquid scintillator to detect $e^{+}$('prompt' signal) and neutron ('delayed' signal). It is surrounded by a layer of pure liquid scintillator to contain gammas, and then a mineral oil 'buffer' layer to separate the photosensors and scintillator. The scintillation light is detected by photomultiplier tubes mounted on the inner wall of the detector.

\section{$2.2 \theta_{13}$ measurement results}
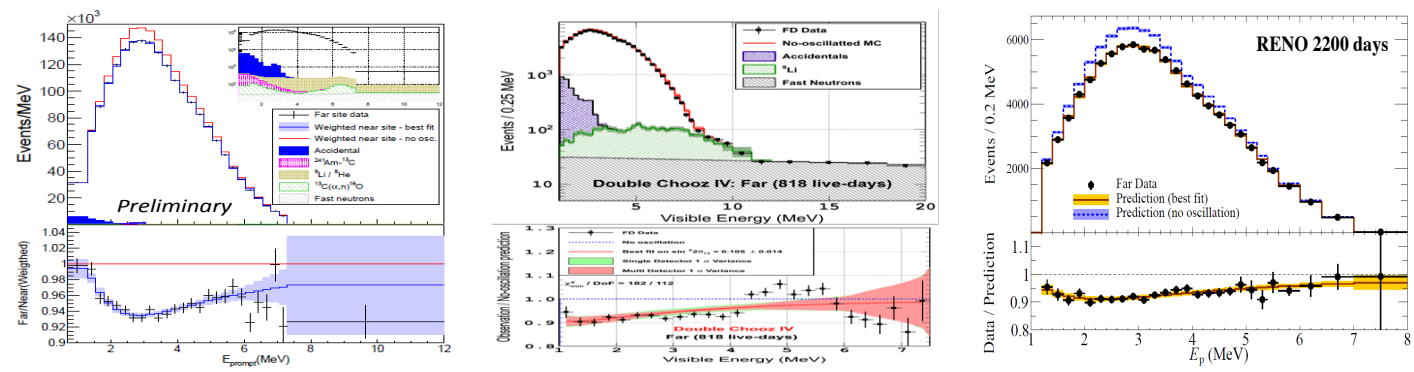

Figure 1: Reconstructed neutrino energy spectra at Daya Bay (left), Double Chooz (middle), and RENO (right) experiments.

Figure 1 shows the reconstructed neutrino energy spectra with far detectors of three experiments. The latest results from three experiments are summarized in Table 2. Although $\theta_{13}$ was the 
Table 2: Results from three reactor experiments

\begin{tabular}{cccc}
\hline \hline Experiment & Daya Bay & Double Chooz & RENO \\
\hline \hline $\sin ^{2} 2 \theta_{13}$ & $0.0856 \pm 0.00429$ & $0.105 \pm 0.014$ & $0.0896 \pm 0.0048 \pm 0.0047$ \\
\hline$\left|\Delta m_{e e}^{2}\right|\left(10^{-3} \mathrm{eV}^{2}\right)$ & $2.52 \pm 0.07$ & & $2.68 \pm 0.12 \pm 0.07$ \\
\hline \hline
\end{tabular}

last mixing angle confirmed to be non-zero, the precision of $\sin ^{2} 2 \theta_{13}$ becomes better than $5 \%$. Now $\theta_{13}$ is measured with the highest precision among three mixing angles. As the three experiments accumulate more data to analyze, we expect even better precision in near future.

\subsection{Future experiment - JUNO}

Jiangmen Underground Neutrino Observatory (JUNO) [5] is designed to determine the mass hierarchy and precisely measure the oscillation parameters. The JUNO detector will be $20 \mathrm{kton}$ liquid scintillator detector at a distance of $53 \mathrm{~km}$ from reactors. The project was approved in 2013 and the civil construction started in 2014. Various detector R\&D and fabrication are in progress, aiming for the start of data taking in 2021.

\section{Accelerator-based long baseline experiments}

For the 'atmospheric' mass-splitting $\left(\Delta m^{2} \simeq 2.5 \times 10^{-3} \mathrm{eV}^{2}\right)$, the first oscillation maximum of neutrinos corresponds to $L / E \simeq 500 \mathrm{~km} / \mathrm{GeV}$. With intense neutrino beams produced at accelerators and gigantic detectors located at hundreds $\mathrm{km}$ of baseline, precise measurements of neutrino oscillations are possible.

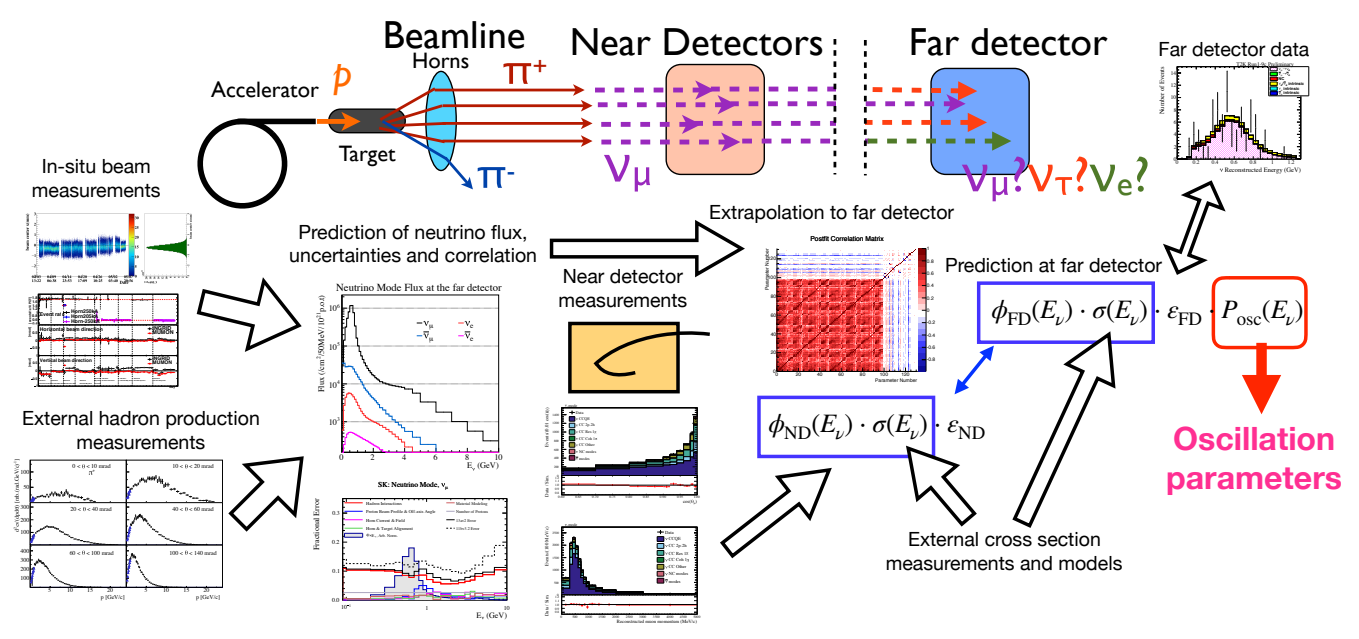

Figure 2: Schematic of a long-baseline experiment

Figure 2 shows a schematic concept of a long-baseline experiment, taking T2K as an example. From the accelerator, almost pure muon neutrino (or muon anti-neutrino, depending on the polarity of the magnetic horns to focus secondary particles) beam is produced. By comparing the 
measurements with near and far detectors, one can measure the muon neutrino disappearance and the electron neutrino appearance. Their oscillation probabilities are written as

$$
\begin{aligned}
P\left(v_{\mu} \rightarrow v_{\mu}\right) & \simeq 1-\left(\cos ^{4} \theta_{13} \sin ^{2} 2 \theta_{23}-\sin ^{2} 2 \theta_{13} \sin ^{2} \theta_{23}\right) \sin ^{2}\left[\frac{\Delta m_{32}^{2} L}{4 E}\right] \\
P\left(v_{\mu} \rightarrow v_{e}\right) & =\sin ^{2} 2 \theta_{13} \sin ^{2} \theta_{23} \frac{\sin ^{2}[(A-1) \Delta]}{(A-1)^{2}} \\
& \mp \alpha \cos \theta_{13} \sin 2 \theta_{12} \sin 2 \theta_{23} \sin 2 \theta_{13} \sin \delta_{\mathrm{CP}} \sin \Delta \frac{\sin A \Delta \sin [(1-A) \Delta]}{A} \\
& +\alpha \cos \theta_{13} \sin 2 \theta_{12} \sin 2 \theta_{23} \sin 2 \theta_{13} \cos \delta_{\mathrm{CP}} \cos \Delta \frac{\sin A \Delta}{A} \frac{\sin [(1-A) \Delta]}{1-A}+O\left(\alpha^{2}\right)
\end{aligned}
$$

where $\alpha=\frac{\Delta m_{21}^{2}}{\Delta m_{32}^{2}} \ll 1, \Delta=\frac{\Delta m_{32}^{2} L}{4 E}, A=2 \sqrt{2} G_{F} N_{e} \frac{E}{\Delta m_{32}^{2}}, G_{F}$ and $N_{e}$ are the Fermi coupling constant and the electron density in matter, respectively. From measurements of these probabilities, $\theta_{23}, \theta_{13}$, $\Delta m_{32}^{2}$, and $\delta_{\mathrm{CP}}$ can be determined.

Table 3: List of long baseline experiments

\begin{tabular}{ccccc}
\hline \hline Accelerator & Experiment & $\begin{array}{c}\text { Baseline } \\
(\mathrm{km})\end{array}$ & $\begin{array}{c}\text { Beam power } \\
(\mathrm{kW})\end{array}$ & Years \\
\hline \hline KEK-PS (KEK) & K2K & 250 & 5 & $1999-2004$ \\
Main Injector (Fermilab) & MINOS(+) & 730 & $400+$ & $2005-2016$ \\
SPS (CERN) & OPERA / ICARUS & 730 & 510 & $2008-2012$ \\
J-PARC MR (J-PARC/KEK) & T2K & 295 & 500 (design:750) & $2009-$ \\
Main Injector (Fermilab) & NOvA & 810 & 700 & $2014-$ \\
\hline \hline
\end{tabular}

Table 3 shows a list of past and present accelerator-based long baseline experiments. The K2K (KEK-to-Kamioka) experiment was the pioneer to establish the technique of the long baseline experiment and confirmed the neutrino oscillation originally observed with atmospheric neutrinos with an artificial neutrino beam. MINOS (later MINOS+ concurrent with NOvA) and OPERA have finished data taking recently and released their final results on the major oscillation measurements. $\mathrm{T} 2 \mathrm{~K}$ and NOvA are taking data and continue to provide new results. In the following, the latest results from these experiments are summarized.

\subsection{Final results from MINOS/MINOS+ and OPERA}

\subsubsection{MINOS/MINOS+}

MINOS and MINOS+ collaborations have recently released the final oscillation results using both neutrino beam and atmospheric neutrinos [6]. Figure 3 shows the reconstructed neutrino energy distribution and the ratio to no-oscillation case with neutrino beam for MINOS and MINOS+ combined data. The data showed consistency with three flavor prediction over a broad range of neutrino energy, which tightly constrains alternate oscillations hypotheses. The measured oscillation parameters are $\left|\Delta m_{32}^{2}\right|=(2.28-2.55) \times 10^{-3} \mathrm{eV}^{2}(\mathrm{NH}),(2.33-2.55) \times 10^{-3} \mathrm{eV}^{2}(\mathrm{IH})$, and $\sin ^{2} \theta_{23}=0.36-0.65$ at $90 \%$ C.L. 

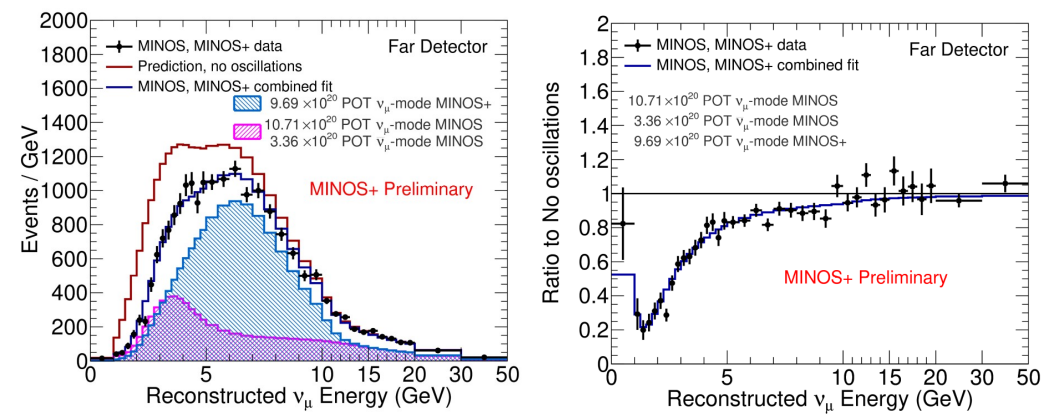

Figure 3: Reconstructed neutrino energy distribution (left) and ratio to no-oscillation case (right) for MINOS/MINOS+ beam data.

\subsubsection{OPERA}

OPERA searched for the $v_{\tau}$ appearance using a beam from CNGS at CERN and detectors located in Gran Sasso, $730 \mathrm{~km}$ away. Figure 4 shows $v_{\tau}$ interaction candidates observed with the emulsion-based detector of OPERA. As the final result, they reported observation of $10 v_{\tau}$ interaction candidates with $2.0 \pm 0.4$ expected background [7]. The $v_{\tau}$ appearance was confirmed with $6.1 \sigma$ significance.
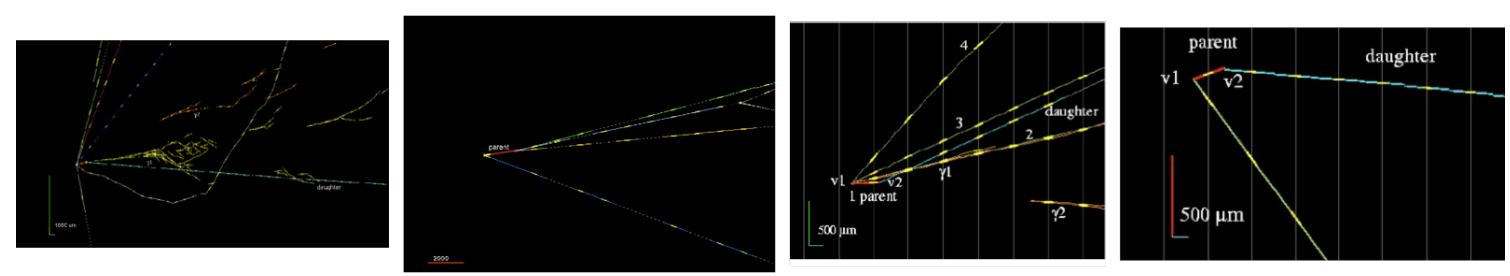

Figure 4: Tau neutrino interaction candidates observed in OPERA experiment.

\subsection{T2K and NOvA}

T2K in Japan and NOvA in US are currently running long baseline experiments. Although two experiments have similar scientific goals, the different baseline lengths result in different amount of matter effect (and possibly others not dependent on L/E), providing complementary information. T2K has a shorter baseline and thus can see purer effect of CP violaion, while NOvA has a longer baseline and more matter effect and sensitivity to the mass hierarchy.

Both experiments released new results in summer 2018. T2K updated their results with 1.5 times more antineutrino data compared to 2017, and NOvA presented their first analysis results including antineutrino beam data.

\subsubsection{T2K results}

T2K uses the Super-Kamiokande detector, a $50 \mathrm{kt}$ water Cherenkov detector, as the far detector. In 2017, T2K applied an improved reconstruction algorithm with a maximum likelihood based on charge and time from all PMTs. It improved signal efficiency and purity, and optimized the fiducial selection to increase statistics by $\sim 20 \%$. The modeling of neutrino-nucleus interaction has been also improved based on the in-situ measurement by near detectors and external data set. 


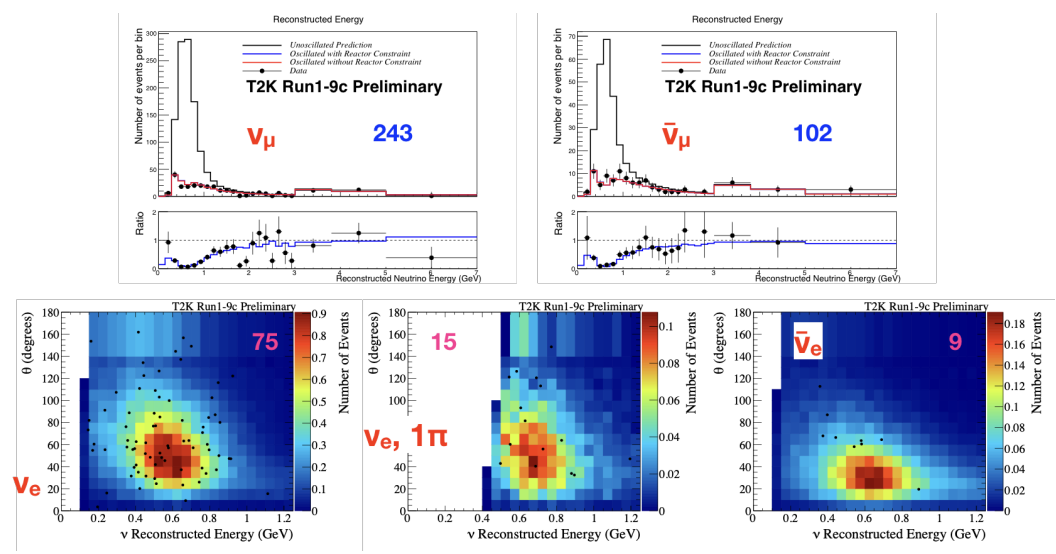

Figure 5: Reconstructed energy distributions for $v_{\mu} / \bar{v}_{\mu}$ (top) and momentum/angular distributions of $e^{-} / e^{+}$ (bottom) of neutrino interaction candidate in neutrino/antineutrino beam with the $\mathrm{T} 2 \mathrm{~K}$ far detector. The number in each plot represents the number of observed events for each category.

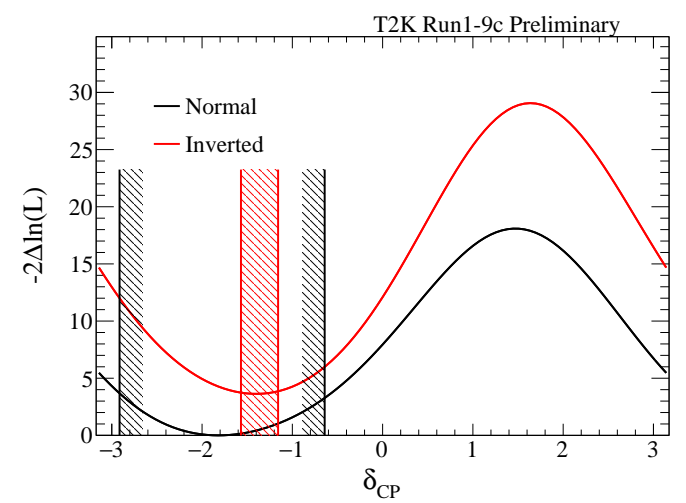

Figure 6: $2 \sigma$ confidence intervals on $\delta_{C P}$ with the T2K data, using $\theta_{13}$ value measured by reactor experiments.

T2K accumulated data corresponding to $3.16 \times 10^{21}$ protons on target (POT) by May 2018 . The results presented here are based on the data taken by December $2017\left(1.49 \times 10^{21}\right.$ POT in neutrino mode and $1.12 \times 10^{21}$ POT in antineutrino mode). Figure 5 shows the distributions of T2K far detector event samples. The neutrino energy is reconstructed from the energy and angle of the observed leptons assuming a two-body interaction. Using those events, $\Delta m^{2}$ and $\theta_{23}$ are measured to be: $\sin ^{2} \theta_{23}=0.536_{-0.046}^{+0.031}$ and $\Delta m_{32}^{2}=(2.434 \pm 0.064) \times 10^{-3} \mathrm{eV}^{2}$ for the normal hierarchy, and $\sin ^{2} \theta_{23}=0.536_{-0.041}^{+0.031}$ and $\Delta m_{13}^{2}=\left(2.410_{-0.063}^{+0.062}\right) \times 10^{-3} \mathrm{eV}^{2}$ for the inverted hierarchy. Posterior probabilities for the mass hierarchy based on a Bayesian analysis are $0.888(0.112)$ for normal (inverted) hierarchy, giving the Bayes factor of 7.9.

A dedicated search for $\bar{v}_{e}$ appearance was performed. The hypothesis with $\bar{v}_{e}$ appearance according to the MNS framework, with parameters constrained with the T2K events other than $\bar{v}_{e}$ sample, and hypothesis with no $\bar{v}_{e}$ appearance were tested against each other. Using both the event rate and momentum/angular distribution of $\bar{v}_{e}$ sample, p-value for the appearance hypothesis is found to be 0.0867 , while that for the no appearance hypothesis is 0.233 .

The CP phase in the lepton sector, $\delta_{C P}$, is constrained by $\mathrm{T} 2 \mathrm{~K}$ data assuming the standard three 

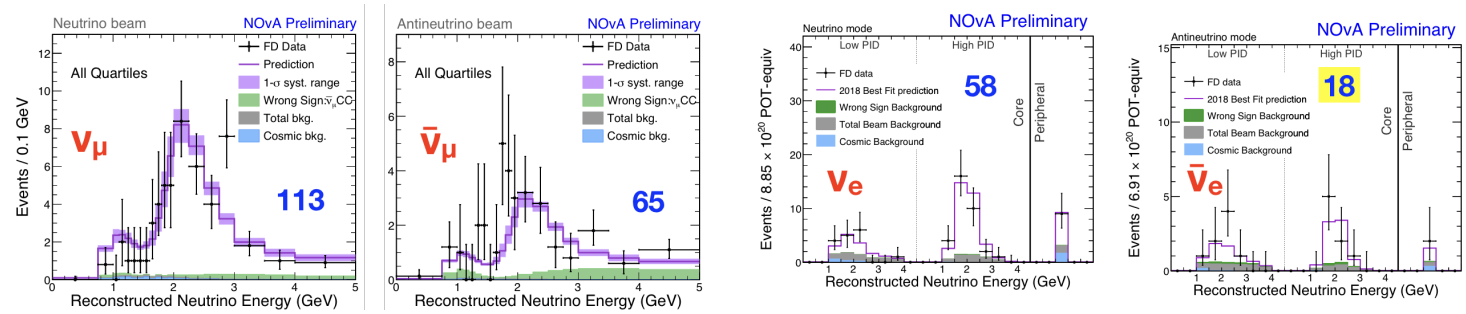

Figure 7: Reconstructed energy distributions for each flavor and neutrino/antineutrino beam with the NOvA far detector. The number in each plot represents the number of observed events for each category.

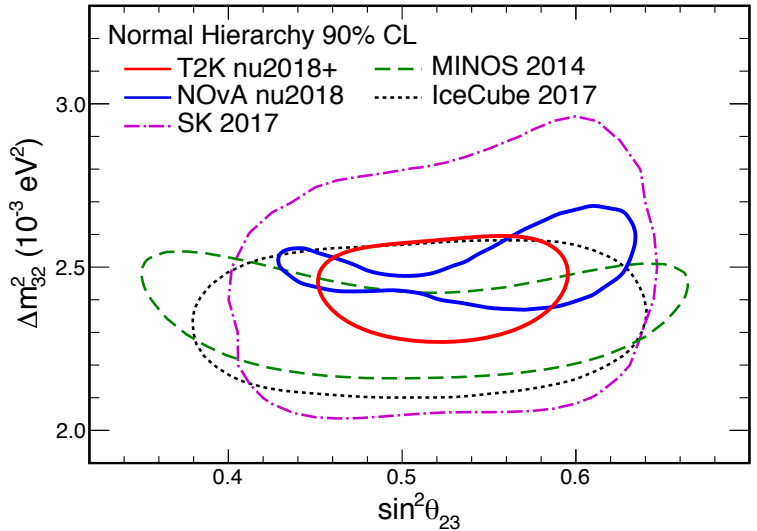

Figure 8: $\sin ^{2} \theta_{23}$ and $\Delta m_{32}^{2}$ measured by T2K, NOvA, Super-Kamiokande, MINOS, and IceCube.

flavor mixing. Figure 6 shows the $2 \sigma$ confidence intervals on $\delta_{C P}$ for each mass hierarchy case. The values with CP conserving case, $\sin \delta_{C P}=0(\delta=0, \pi)$, are outside of $2 \sigma$ confidence intervals. $\mathrm{T} 2 \mathrm{~K}$ has provided the first hint of $\mathrm{CP}$ violation in the lepton sector.

\subsubsection{NOvA results}

The NOvA near and far detectors are scintillating tracker-calorimeters consisting of layered PVC cells filled with liquid scintillator. NOvA pioneered the use of deep-learning technique in neutrino experiment; Convolutional Neural Networks are used for particle identification and event classification. They improved classifier used for 2018 analysis with separate training for the neutrino and antineutrino beams. The neutrino energy is reconstructed by adding the lepton energy (reconstructed from range for $\mu$, calorimetric measurement for $e$ ) and hadron energy with calorimetric measurement.

Figure 7 shows the reconstructed energy distributions for each flavor and neutrino/antineutrino beam with the NOvA far detector, with data corresponding to $8.85 \times 10^{20}$ POT with neutrino and $6.9 \times 10^{20}$ POT with antineutrino beams. NOvA observed $18 \bar{v}_{e}$ candidates, where the expected background is 4.7-5.7 events. With this first set of antineutrino data, they have found the evidence of $\bar{v}_{e}$ appearance with $>4 \sigma$ significance. From a joint fit of all samples, they measure $\sin ^{2} \theta_{23}=$ $0.58 \pm 0.03$ and $\Delta m_{32}^{2}=\left(2.51_{-0.08}^{+0.12}\right) \times 10^{-3} \mathrm{eV}^{2}$. NOvA data prefer the non-maximal value $\left(\theta_{23} \neq\right.$ $45^{\circ}$ at $1.8 \sigma$, and also higher octant at a similar level. 
Figure 8 shows the comparison of $\sin ^{2} \theta_{23}$ and $\Delta m_{32}^{2}$ measured by various experiments. Currently, all results are consistent with each other and also consistent with the maximal $\theta_{23}$ mixing.

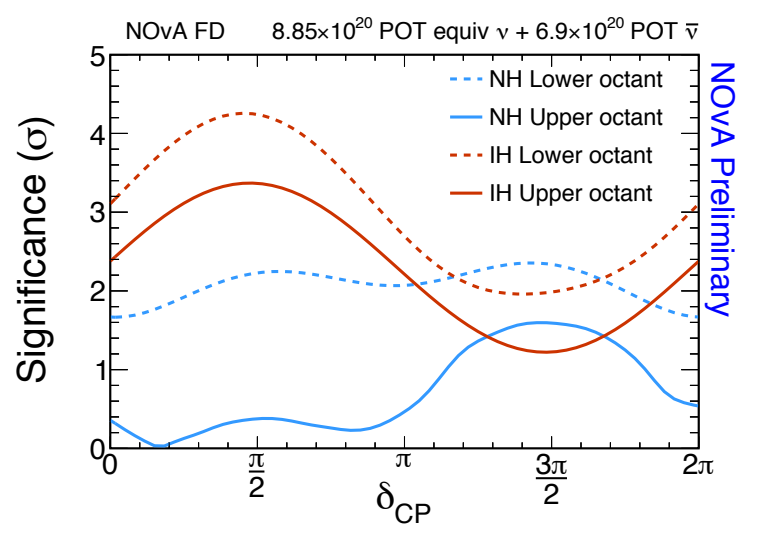

Figure 9: $\delta_{C P}$ constraints from NOvA.

Figure 9 shows the NOvA result on the constraint on $\delta_{C P}$. The best fit point is found at $\delta_{C P}=0.17 \pi$ and normal hierarchy, although all the values are within about $1 \sigma$ for the normal hierarchy case.

\subsection{Future prospects}

Figure 10 shows so-called bi-event plots for T2K and NOvA. They compare the number of observed events for neutrino and anti-neutrino modes, with predictions for normal and inverted hierarchies and various mixing parameters. It can be seen that $\mathrm{T} 2 \mathrm{~K}$ and NOvA have started to constrain the significant part of the parameter space, which shows that we are at the beginning of the era of the measurement of $\mathrm{CP}$ violation in the lepton sector. Clearly, on the other hand, both experiments need more data and improved sensitivity for definite measurements.
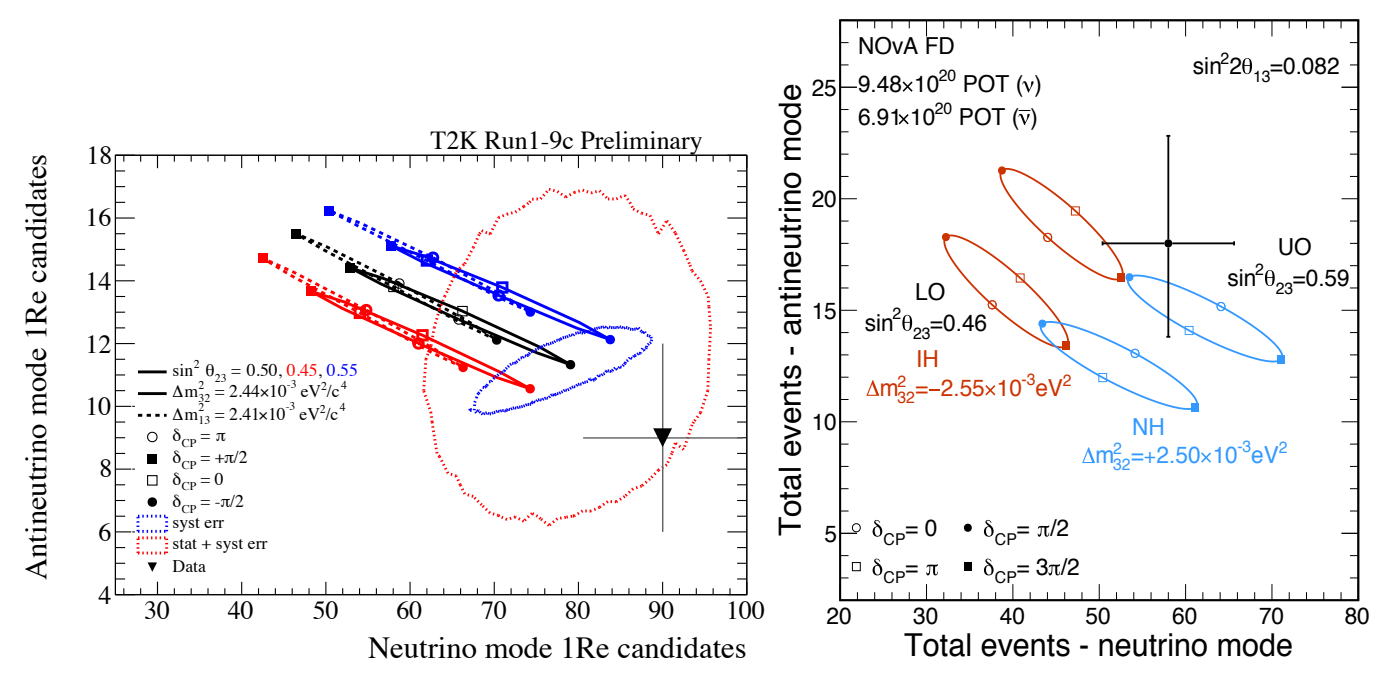

Figure 10: Bi-event plot for T2K (left) and NOvA (right). 
Both experiments envision to further enhance their capabilities. T2K plans to increase the beam power to $1.3 \mathrm{MW}$ and upgrade the beamline and near detectors. NOvA plans also accelerator improvement to $0.9 \mathrm{MW}$ beam power, as well as a test beam to improve the detector systematic uncertainties. With those efforts in addition to analysis improvements, we have very good prospects for determination of mass hierarchy and search for (and measurement of CP violation).

Beyond the current experiments, there are future experiments planned in the next decade, Hyper-Kamiokande [10] and DUNE [11]. The neutrino oscillation studies will continue with these large international projects, as explained elsewhere [12].

\section{Conclusions}

With reactor and accelerator experiments playing key roles, there have been rapid and steady progress in neutrino oscillation physics for the past tow decades. In this contribution, the latest results from those experiments are presented. Daya Bay/Double Chooz/RENO continue to improve precision of $\theta_{13}$, while JUNO is coming online in near future. The final results from MINOS/MINOS+ and OPERA strongly support the three flavor mixing scenario. T2K and NOvA started to explore $\mathrm{CP}$ violation in the lepton sector and mass hierarchy. T2K has reported a hint of CP violation. NOvA reported an evidence for $\bar{v}_{e}$ appearance with $>4 \sigma$ significance with their first antineutrino data. They will continue to provide interesting results with more neutrino and antineutrino beam and possible upgrade to the experimental configuration. The area of neutrino oscillation will stay a very exciting field for decades to come.

\section{References}

[1] Y. Fukuda et al. [Super-Kamiokande Collaboration], Phys. Rev. Lett. 81 (1998) 1562.

[2] D. Adey et al. [Daya Bay Collaboration], Phys. Rev. Lett. 121, 241805 (2018). See also L. Zhan, these proceedings.

[3] A. Stahl, these proceedings.

[4] G. Bak et al. [RENO Collaboration], Phys. Rev. Lett. 121, 201801 (2018). See also H. K. Seo, these proceedings.

[5] Q. Zhang, these proceedings.

[6] A. Aurisano, talk given at NEUTRINO2018, DOI:10.5281/zenodo.1286760.

[7] N. Agafonova et al. [OPERA Collaboration], Phys. Rev. Lett. 120, no. 21, 211801 (2018) Erratum: [Phys. Rev. Lett. 121, no. 13, 139901 (2018)].

[8] M. O. Wascko, talk given at NEUTRINO2018, DOI:10.5281/zenodo.1286752. See also P. Litchfield, these proceedings.

[9] M. Sanchez, talk given at NEUTRINO2018, DOI:10.5281/zenodo.1286757. See also J. Bian, these proceedings.

[10] K. Abe et al., arXiv:1109.3262 [hep-ex]; K. Abe et al. [Hyper-Kamiokande Collaboration], arXiv: 1805.04163 [physics.ins-det].

[11] R. Acciarri et al. [DUNE Collaboration], arXiv:1512.06148 [physics.ins-det].

[12] J. Yu, these proceedings. 